\title{
Sexual Behavior in an Intensive Care Unit
}

\author{
Dimitrios Tsamis • Dimitrios Theodorou • \\ Stylianos Katsaragakis
}

Published online: 8 March 2012

(c) Springer Science+Business Media, LLC 2012

Sexuality is something which is intimately combined with the human life and behavior. But in cases in which the human body is severely ill, the sexual instincts are sidelined and subsided. Conversely, fears are raised about survival and condition of health. In intensive care units (ICU), sexuality is most of the times absent. But there are some cases where sexual desire is present and is intense (Kotronoulas, Papadopoulou, \& Patiraki, 2009; Wilmoth, 2007). We report two cases of patients who demonstrated intense sexual desire by masturbating in the area of an ICU.

The first patient was a 26-year-old male, who after eight gunshots and two surgical interventions for massive bleeding from liver parenchyma was stabilized and withdrawn from mechanical ventilation. The patient about 3 days before leaving ICU performed masturbation, even if a urinary catheter was present. The overall stay of the patient in the ICU was approximately 3 months.

The second patient was a 55-year-old female patient. This woman was a morbidly obese patient who underwent a sleeve gastrectomy. At the third postoperative day, sepsis was developed since a leak from the stapling line near the cardia of the stomach was present. The patient underwent surgical drainage of the region and cervical esophagostomy was performed. During the monitoring of the patient in the ICU, she underwent masturbation. The overall stay of the patient in the ICU was 40 days and the incident was performed 35 days approximately after the transfer of the patient at the ICU.

This phenomenon is rare in patients who are critically ill and are hospitalized in an ICU. Nevertheless, both of these patients were in relatively good overall condition near their export from the unit. Thus, both of these patients had a long stay with nonexistent sexual activity. Their medication in the ICU had not diminished sexual desire as an adverse effect. Maybe this act and the return of the sexual desire were indicative of the good clinical condition of the patients and the overtaking of their medical problems. It is very interesting the fact that this instinct was greater than awkwardness which the patients may felt by the presence of the personnel of ICU.

\section{References}

Kotronoulas, G., Papadopoulou, C., \& Patiraki, E. (2009). Nurses' knowledge, attitudes, and practices regarding provision of sexual health care in patients with cancer: Critical review of the evidence. Supportive Care in Cancer, 17, 479-501.

Wilmoth, M.C. (2007). Sexuality: A critical component of quality of life in chronic disease. Nursing Clinics of North America, 42, 507-514.

D. Tsamis $\cdot$ D. Theodorou $\cdot$ S. Katsaragakis

Intensive Care Unit of Surgical Trauma, 1st Department of

Propaedeutic Surgery, Hippokrateion General Hospital, Athens

Medical School, University of Athens, Athens, Greece

D. Tsamis ( $\square)$

7 Kalvou Street, 11473 Athens, Greece

e-mail: tsamisdim@hotmail.com 